\title{
Convergence in Dual Space
}

\author{
Amar nath kumar, Brij Nandan Prasad \\ Head,Dept. of Mathematics,Deo Sharan Women's Evening College, Sohsarai, Magadh University, India \\ Head, Dept. of Mathematics, Nalanda College, Biharsharif, Magadh University, India
}

\begin{abstract}
In this paper we have deal with the role of a dual space of a function space. In dealing so we will try to observe that the behavior of the dual space is the same as for the function space or not. That is why if a result holds good for a function space then same type of result also holds good for it dual space in case of convergences.
\end{abstract}

Keywords: Linear space, Sequence space, Function space, Dual Space, Perfect space, Parametric limit and parametric convergent, projective limit and projective convergent.

\section{Introduction}

Enormous work had been done on dual space of a sequence space . Also a massive corpus of work has been done in convergences of different kinds of sequence and sequence spaces. An account of all these can be found out in Cook,1, chapter 10 P.272-326 . Later on dual space was extended to the case of function spaces . In this concern convergences has also been extended to the case of function spaces by some students of the school of mathematics. The role of dual space of a function space was also observed by some of the researcher of this field in the study of different discipline .

In this paper we have used the notion of dual space of a function space to study the behaviour of convergences in different suitably defined function spaces. That is we took the pain to establish some of the results on different kind of convergences for function spaces using the notion of a dual space of a function space . Moreover in course of extending some of the results we tried and got success in observing that if a result is true for a particular function space then it also stands good for another some of the function spaces.

The object of this paper is in fact to establish some of the results to show that parametric convergence implies projective convergence in the dual space of a function space.

\section{For this section we refer to definitions of some essential special function spaces as preliminaries, making the use of which some results have been established. Moreover, The integration has been taken through in Lebesgue sense in the interval $[0, \square)$. We denote the set $[0, \square)$ by $\mathbf{E}$.}

2.1Sequence Space : A linear space whose elements are sequences is called a sequence space .Thus a set $\mathrm{V}$ of sequences is a sequence space if, it contains the origin and for every $\mathrm{x} . \mathrm{y}$ in $\mathrm{V}$ and for every scalar $\alpha, \mathrm{x}+\mathrm{y}$ and $\alpha \mathrm{x}$ are in $\mathrm{V}$.

2.2Function Space :A linear space whose elements are functions is called a function space.Thus set $\mathrm{V}$ of functions is a functions space if it contains the origin and for $f, g$ in $V$ and for every scalar $\alpha, f+g$ and $\alpha$ are in V.Here we consider only real functions of real variables so $\alpha$ is taken to be real scalar so that our purpose is served .

Moreover, the integration has been taken through in Lebesgue sense in the interval $[0, \infty)$. We denote the set $[0, \infty)$ by $\mathrm{E}$.

2.2.1 $\Gamma$ : It denotes the space of all convergent and bounded functions.

2.2.2 $\mathrm{L}_{\infty}$ : It denotes the space of all functions $\mathrm{f}$ such that $|\mathrm{f}(\mathrm{x})|<\mathrm{K}$ for almost all $\mathrm{x} \geq 0$ where $\mathrm{K}$ is constant .

2.2.3 $\mathrm{L}_{1}$ : It denotes the space of integrable functions, that is $\mathrm{L}_{1}$ is the space of all functions $\mathrm{f}$ such that $\int_{\mathrm{E}}|\mathrm{f}(\mathrm{x})| \mathrm{dx}<\infty$

2.2.4 $\zeta$ : It denotes the space of all functions continuous and bounded in $[0, \infty)$. Clearly $\zeta<\mathrm{L}_{\infty}$

2.3 Dual Space of a function space : Let $\alpha$ be a function then the dual space of $\alpha$ is denoted by $\alpha^{*}$ and is defined to be the space of all functions $f$ such that $\int_{E}|f(x) g(x)| d x<\infty$ for every function $g$ in $\alpha$.

Also $\alpha^{*}$, the dual space of $\alpha$, is again a function space.

(we refer to Ph.D. Thesis M.U.Bodhgaya,1985 by L.K.Sharan ).

2.4 Perfect function Space : A function space $\alpha$ is said to be perfect when $\alpha^{* *}=\alpha$.

2.5 Parametric Convergent (or $t$ - convergent ) :Let $\mathrm{f}_{\mathrm{t}}(\mathrm{x})$ be a family of functions of $\mathrm{x}$ defined for all $\mathrm{t}$ in $[0, \infty)$ , where $t$ is a parameter .If to every $\epsilon>0$, there corresponds a positive number $T(\epsilon)$, independent of $x$, such 
that , for almost all $\mathrm{x} \geq 0,\left|\mathrm{f}_{\mathrm{t}}(\mathrm{x})-\mathrm{f}_{\mathrm{t}}^{1}(\mathrm{x})\right| \leq \epsilon$ for all $\mathrm{t}, \mathrm{t}^{1} \geq \mathrm{T}(\epsilon)$, then the family $\mathrm{f}_{\mathrm{t}}(\mathrm{x})$ is said to be parametric convergent $(\mathrm{t}-\mathrm{cgt})$.

2.6 Projective Convergence ( or $\alpha \beta$-convergence or p-convergence ) : Let $\alpha^{*} \supseteq \beta$ and

$F_{g}(t)=\int_{E} f_{t}(x) g(x) d x$. Where $f_{t}(x)$ is in $\alpha$ ang $g(x)$ is in $\beta$ then if $F_{g}(t)$ tends to a definite finite limit as $t-$ tends to $\infty$ for every $g(x)$ in $\beta$ then we say that $f_{t}(x)$ is projective convergent ( or p-convergent) relative to $\beta$, or $f_{t}(x)$ is $\alpha \beta$-convergent and $f_{t}(x)$ is simply called $p$-convergent in $\alpha$ or $\alpha$-convergent when $\beta=\alpha^{*}$.

2.6.1A necessary and sufficient condition for $\alpha \beta$ - convergence of $\mathrm{f}_{\mathrm{t}}(\mathrm{x})$ is that to every $\mathrm{g}$ in $\beta$ and to every $\epsilon>0$, there corresponds a positive number $T(\epsilon, g)$ such that, for all $t, t^{1} \geq T(\epsilon, g),\left|\int_{E} g(x)\left\{f_{t}(x)-f_{t^{1}}(x)\right\} d x\right| \leq \epsilon$.

\section{In this section we establish some of the results with reference of the notions given in the} above section.

Theorem (3.1): If a family of functions in a function space $\Gamma^{* *}$ be parametric convergent then it is $\Gamma^{* *} \Gamma^{*}$ convergent .

Proof : Let $f_{t}$ be a family of functions in function space $\Gamma^{* *} \Gamma^{*}$. Also let $f_{t}$ be parametric convergent in $\Gamma^{* * *} \Gamma^{*}$.Then to every $\epsilon>0$, there exists a positive number $\mathrm{T}(\epsilon)$, independent of $\mathrm{x}$, such that , for almost all $\mathrm{x}$ $\geq 0,\left|\mathbf{f}_{\mathbf{t}}(\mathbf{x})-\mathbf{f}_{\mathrm{t}}^{1}(\mathbf{x})\right| \square \boldsymbol{\epsilon} \quad \ldots \ldots \ldots \ldots \ldots \ldots . .(2.11)$ for all $\mathrm{t}, \mathrm{t}^{1} \geq \mathrm{T}(\epsilon)$, Now let $\mathrm{g}(\mathrm{x})$ be any function in $\Gamma^{*}$. Hence $\mathrm{g}(\mathrm{x})$ must be in $\mathrm{L}_{1}$ Thus $\int_{\mathrm{E}}|\mathbf{g}(\mathbf{x})| \mathbf{d x}<$

Now since we see that,$\left|\int_{\mathrm{E}} \mathbf{g}(\mathbf{x})\left\{\mathbf{f}_{\mathbf{t}}(\mathbf{x})-\mathbf{f}_{\mathbf{t}^{1}}(\mathbf{x})\right\} \mathbf{d x}\right| \square \int_{\mathbf{E}}\left|\mathbf{g}(\mathbf{x})\left\{\mathbf{f}_{\mathbf{t}}(\mathbf{x})-\mathbf{f}_{\mathbf{t}^{1}}(\mathbf{x})\right\}\right| \mathbf{d x}$

$\square \boldsymbol{\epsilon} \int_{\mathbf{E}}|\mathbf{g}(\mathbf{x})| \mathbf{d x} \quad\left[\right.$ By (2.11)] $\square \boldsymbol{\epsilon} \mathbf{K}(\mathbf{g})$ [By (2.12)] for all $\mathrm{t}, \mathrm{t}^{1} \geq \mathrm{T}(\epsilon)$ every $\epsilon>0$, where $\mathrm{K}(\mathrm{g})$ is a constant depending on $\mathrm{g}$ but independent of $\mathrm{t}$ in $\mathrm{E}=[0, \infty)$. But then by the necessary and sufficient conditions for $f_{t}$ to be $\Gamma^{* * *} \Gamma^{*}$-convergent. $f_{t}$ is $\Gamma^{* * *} \Gamma^{*}$-convergent .Or simply $f_{t}$ is $\Gamma^{* *} \Gamma^{*}$-convergent .

Theorem (3.2,) : In theorem $(2.3, I)$ if $\Gamma$ is perfect then the family $f_{t}$ of functions in $\Gamma$ is $\Gamma^{* *} \Gamma^{*}$-convergent in the case $\mathrm{f}_{\mathrm{t}}$ is parametric convergent .

Proof : Since $f_{t}$, a family of functions, is in $\Gamma^{* *}$. But by hypothesis $\Gamma$ is perfect Thus $\Gamma^{* *}=\Gamma$ Hence $f_{t}$ is in $\Gamma^{* *}$ implies that $f_{t}$ is in $\Gamma$.Also $f_{t}$ is parametric convergent in $\Gamma^{* *}$ so $f_{t}$ is parametric convergent in $\Gamma$. Hence then to every $\epsilon>0$, there exists a positive number $\mathrm{T}(\epsilon)$, independent of $\mathrm{x}$, such that , for almost all $\mathrm{x} \geq 0$, $\mathbf{f}_{\mathbf{t}}(\mathbf{x})-\mathbf{f}_{\mathbf{t}} \mathbf{1}(\mathbf{x}) \mid \square \boldsymbol{\epsilon} \quad \ldots \ldots \ldots \ldots \ldots \ldots(2.13)$

for all $t, t^{1} \geq T(\epsilon)$, Now if $g(x)$ be any function in $\Gamma^{*}$. Then $g(x)$ must be in $L_{1}$

Hence $\int_{\mathbf{E}}|\mathbf{g}(\mathbf{x})| \mathbf{d} \mathbf{x}=\int_{\mathbf{0}}^{\infty}|\mathbf{g}(\mathbf{x})| \mathbf{d x}<$

Now , since, $\left|\int_{\mathbf{E}} \mathbf{g}(\mathbf{x})\left\{\mathbf{f}_{\mathbf{t}}(\mathbf{x})-\mathbf{f}_{\mathbf{t}^{1}}(\mathbf{x})\right\} \mathbf{d} \mathbf{x}\right| \square \quad \int_{\mathbf{E}}\left|\mathbf{g}(\mathbf{x})\left\{\mathbf{f}_{\mathbf{t}}(\mathbf{x})-\mathbf{f}_{\mathbf{t}^{1}}(\mathbf{x})\right\}\right| \mathbf{d x} \quad \square \boldsymbol{\epsilon} \int_{\mathbf{E}}|\mathbf{g}(\mathbf{x})| \mathbf{d x}[\mathrm{By}(2.13)] \square \boldsymbol{\epsilon}$ $\mathbf{K}(\mathbf{g})$ [By (2.14)] for all $\mathrm{t}, \mathrm{t}^{1} \geq \mathrm{T}(\epsilon)$ every $\epsilon>0$, where $\mathrm{K}(\mathrm{g})$ is a constant depending on $\mathrm{g}$ but independent of $t$ in $E=[0, \infty)$. But then by the necessary and sufficient conditions for a family $f_{t}$ of functions to be projective convergent we find that $f_{t}$ is $\Gamma^{* * *} \Gamma^{*}$-convergent. Or simply $f_{t}$ is $\Gamma^{* *} \Gamma^{*}$-convergent .

Theorem (3.3) : Let $f_{t}$ be a family of functions in $L_{\infty}^{* *}$ which is parametric convergent then it is projective convergent

Proof : Let $f_{t}$ be a family of functions of $x$ in $L_{\infty}^{* *}$ defined for all $t$ in $[0, \infty)$, where $t$ is parameter, By hypothesis $\mathrm{f}_{\mathrm{t}}$ is parametric convergent then to every $\epsilon>0$, there exists a positive number $\mathrm{T}(\epsilon)$, independent of $\mathrm{x}$, such that, for almost all $\mathrm{x} \geq 0,\left|\mathbf{f}_{\mathbf{t}}(\mathbf{x})-\mathbf{f}_{\mathrm{t}}^{1}(\mathbf{x})\right| \square \boldsymbol{\epsilon}$

for all $t, t^{1} \geq T(\epsilon)$, Now let $g(x)$ be any function in $L_{\infty}^{*}$. But the dual space of $L_{\infty}$ is always contained in $\mathrm{L}_{1}$. Hence $\mathrm{g}(\mathrm{x}) \in \mathrm{L}_{\infty}^{*}$ implies $\mathrm{g}(\mathrm{x})$ must be in $\mathrm{L}_{1}$ but then, $\int_{\mathbf{E}}|\mathbf{g}(\mathbf{x})| \mathbf{d x}<\square \ldots \ldots(2.16)$

We have to show that $f_{t}$ is $L_{\infty}^{* *} L_{\infty}^{*}$-convergent That is to show that to every $g(x)$ in $L_{\infty}^{*}$ and to every $\epsilon>0$, there corresponds a positive number $\mathrm{T}(\epsilon, \mathrm{g})$ such that , for all $\mathrm{t}, \mathrm{t}^{1} \geq \mathrm{T}(\epsilon, \mathrm{g})\left|\int_{\mathbf{E}} \mathbf{g}(\mathbf{x})\left\{\mathbf{f}_{\mathbf{t}}(\mathbf{x})-\mathbf{f}_{\mathbf{t}^{1}}(\mathbf{x})\right\} \mathbf{d x}\right|$ $\boldsymbol{\epsilon}$, Now since, $\left|\int_{E} \mathbf{g}(\mathbf{x})\left\{\mathbf{f}_{\mathbf{t}}(\mathbf{x})-\mathbf{f}_{\mathbf{t}^{1}}(\mathbf{x})\right\} \mathbf{d x}\right| \square\left|\int_{\mathbf{E}}\right| \mathbf{g}(\mathbf{x})\left\{\mathbf{f}_{\mathbf{t}}(\mathbf{x})-\mathbf{f}_{\mathbf{t}^{1}}(\mathbf{x})\right\}\left|\mathbf{d x} \quad \square \boldsymbol{\epsilon} \int_{E}\right| \mathbf{g}(\mathbf{x}) \mid \mathbf{d x}[$ By (2.15)] $\square$ $\boldsymbol{\epsilon} \mathbf{K}(\mathbf{g})$ [By (2.16)] for all $\mathrm{t}, \mathrm{t}^{1} \geq \mathrm{T}(\epsilon)$ every $\epsilon>0$, where $\mathrm{K}(\mathrm{g})$ is a constant depending on $\mathrm{g}$ but independent of $t$ in $E=[0, \infty)$.Thus $f_{t}(x)$ is $L_{\infty}^{* *} L_{\infty}^{*}$-convergent .Or simply without any scope of confusion $\mathrm{f}_{\mathrm{t}}(\mathrm{x})$ is $\mathrm{L}_{\infty}^{* *}$ - convergent .However if $\mathrm{L}_{\infty}$ is perfect then $\mathrm{L}_{\infty}^{* *}=\mathrm{L}_{\infty}$ and then the above theorem can be restated as Theorem (3.4) : Every parametric convergent family $f_{t}(x)$ of functions of $x$ in $L_{\infty}$ is $L_{\infty}$ - convergent .

Proof : We can prove this theorem the line of proof of the just above theorem .Clearly $\mathrm{f}_{\mathrm{t}}(\mathrm{x})$ is in $\mathrm{L}_{\infty}$ and is parametric convergent so it does not matter that whether $\mathrm{f}_{t}(\mathrm{x})$ is in $\mathrm{L}_{\infty}^{* *}$ or $\mathrm{L}_{\infty}$ as $\mathrm{L}_{\infty}$ is supposed to be perfect . Again it follows direct from the definition of parametric convergent of $\mathrm{f}_{\mathrm{t}}(\mathrm{x})$ in any function space $\alpha$ whether if $\alpha=\mathrm{L}_{\infty}$ that to every $\epsilon>0$, there corresponds a positive number $\mathrm{T}(\epsilon)$, independent of $\mathrm{x}$ such that , for almost all $\mathrm{x} \geq 0 .\left|\mathbf{f}_{\mathbf{t}}(\mathbf{x})-\mathbf{f}_{\mathrm{t}}^{1}(\mathbf{x})\right| \square \boldsymbol{\epsilon} \quad \ldots \ldots \ldots \ldots \ldots . . .(2.17)$

for all $t, t^{1} \geq T(\epsilon)$, Now in order to prove that $f_{t}(x)$ is $L_{\infty} L_{\infty}^{*}$ - convergent . 
We need a function $\mathrm{g}(\mathrm{x})$ in $\mathrm{L}_{\infty}^{*}$ so as before in the previous theorem $(2.3, \mathrm{III}) \mathrm{g}(\mathrm{x})$ in $\mathrm{L}_{\infty}^{*}$ implies $\mathrm{g}(\mathrm{x})$ is in $\mathrm{L}_{1}$ because $\mathrm{L}_{1}$ is the dual space of $\mathrm{L}_{\infty}$. Hence $\mathrm{L}_{\infty}^{*}=\mathrm{L}_{1}$. Thus $\mathrm{g}(\mathrm{x}) \in \mathrm{L}_{\infty}^{*} \Rightarrow \mathrm{g}(\mathrm{x})$ is in $\mathrm{L}_{1}$.

So again we shall have that $\int_{\mathbf{E}}|\mathbf{g}(\mathbf{x})| \mathbf{d x}<$

Now with theorem help of $[(2.17)$ and (2.18)] we can see that

$\left|\int_{E} \mathbf{g}(\mathbf{x})\left\{\mathbf{f}_{\mathbf{t}}(\mathbf{x})-\mathbf{f}_{\mathbf{t}^{1}}(\mathbf{x})\right\} \mathbf{d x}\right| \square\left|\int_{\mathrm{E}}\right| \mathbf{g}(\mathbf{x})\left\{\mathbf{f}_{\mathbf{t}}(\mathbf{x})-\mathbf{f}_{\mathbf{t}^{\mathbf{1}}}(\mathbf{x})\right\}\left|\mathbf{d x} \square \epsilon \int_{\mathrm{E}}\right| \mathbf{g}(\mathbf{x}) \mid \mathbf{d x} \square \boldsymbol{\epsilon} \mathbf{K}(\mathbf{g})$

for all $\mathrm{t}, \mathrm{t}^{1} \geq \mathrm{T}(\epsilon)$ every $\epsilon>0$, where $\mathrm{K}(\mathrm{g})$ is a constant depending on $\mathrm{g}$ but independent of theorem parameter $t$ in $E=[0, \infty)$.Thus $f_{t}(x)$ is $L_{\infty} L_{\infty}^{*}$ - convergent . Or $f_{t}(x)$ is $L_{\infty}$-convergent .

Theorem (3.5) :In $\square^{* * *}$ parametric convergent implies $\square^{* *} \square^{*}$ - convergent .

Proof : Let $f_{t}(x)$ be a family of functions of $x$ in $\square^{* *}$ defined for all $t$ in $[0, \infty)$, where $t$ is a parameter. We now suppose that the family $f_{t}$ is parametric convergent but then, by definition, to every $\epsilon>0$, there exists a positive number $T(\epsilon)$, independent of $x$ such that, for almost all $x \geq 0$

$$
\left|\mathbf{f}_{\mathbf{t}}(\mathbf{x})-\mathbf{f}_{\mathbf{t}}{ }^{1}(\mathbf{x})\right| \square \boldsymbol{\epsilon} \quad \ldots \ldots \ldots \ldots . . .(2.19) \text { for all } \mathrm{t}, \mathrm{t}^{1} \geq \mathrm{T}(\epsilon)
$$

Let $\mathrm{g}(\mathrm{x})$ be any function in $\square^{*}$ then $\mathrm{g}(\mathrm{x})$ will get itself into theorem space of integrable functions, that is $\mathrm{g}(\mathrm{x})$ will be such that $\int_{\mathbf{E}}|\mathbf{g}(\mathbf{x})| \mathbf{d x}<$

Now on the basis of [(2.19) and (2.20)] it is easy to see that $\left|\int_{\mathbf{E}} \mathbf{g}(\mathbf{x})\left\{\mathbf{f}_{\mathbf{t}}(\mathbf{x})-\mathbf{f}_{\mathbf{t}^{1}}(\mathbf{x})\right\} \mathbf{d} \mathbf{x}\right|$

$\square\left|\int_{\mathbf{E}}\right| \mathbf{g}(\mathbf{x})\left\{\mathbf{f}_{\mathbf{t}}(\mathbf{x})-\mathbf{f}_{\mathbf{t}^{\mathbf{1}}}(\mathbf{x})\right\}\left|\mathbf{d x} \square \boldsymbol{\epsilon} \int_{\mathbf{E}}\right| \mathbf{g}(\mathbf{x}) \mid \mathbf{d x}[$ By (2.19)] $\square \boldsymbol{\epsilon} \mathbf{K}(\mathbf{g})$

for all $\mathrm{t}, \mathrm{t}^{1} \geq \mathrm{T}(\epsilon)$ every $\epsilon>0$, where $\mathrm{K}(\mathrm{g})$ is a constant depending on $\mathrm{g}$ but independent of theorem parameter $\mathrm{t}$ in $\mathrm{E}=[0, \infty)$. But then by a necessary and sufficient condition parametric convergent family of functions $\mathrm{f}_{\mathrm{t}}(\mathrm{x})$ defined for all $\mathrm{x}$ is $\square^{* *} \square^{*}$ - convergent .

Corollary 1. If in the above theorem $\square$ is perfect then $\square^{* *}=\square$

But then it is immediate deduction that parametric convergent of family $\mathrm{f}_{\mathrm{t}}(\mathrm{x})$ of functions defined for all $\mathrm{x}$ and all $\mathrm{t}$ in $\mathrm{E}$, where $\mathrm{t}$ is a parameter, is $\square \square^{*}$-convergent .

The proof follows exactly to the cases when the function spaces $\Gamma$ and $\mathrm{L}_{\infty}$ are perfect.

\section{Conclusion}

We have observed that the behavior of the dual space is the same as for the function space. We also observed that the technique of establishing the results for function spaces are quite different from those of the technique of establishing the results to the case of sequence space. As a matter of fact the main reason behind it is the fact that in case of sequence space we had to deal with integers whereas in the case of function or function space, we always play with the continuous functions. On the basis of above result we can suggest all the result for function space also holds good by dual space of function space. Its also extend one step ahead the theory of dual space of function space by different suitably defined function spaces.

\section{Acknowledgement}

We would like to take this opportunity to record our deep sense of gratitude to Prof(Dr.) Lalit Kumar Sharan , Head, Department of Mathematics, Veer Kunwar Singh University ,Ara ,Bihar for his kind guidance.

Our thanks also go to some of my friends who wanted to be unnamed..

Amar Nath Kumar \& Brij Nandan Prasad

\section{Books:}

\section{References}

[1]. ALLEN ,HS:Projective Convergence and Limit in Sequence Spaces , Proc . London Math Soc ., (2) , 48 , (1944), 310-338 . Cited by 12

[2]. COOKE ,R .G.:Infinite Matrices and Sequence Spaces , macmillan , London , (1950). Cited by 626

[3]. HARDY, G.H .:Divergent Series, Oxford, (1956). Cited by 4087

[4]. JHA, K.K. : Advanced Set Theory and The Foundations of Mathematics, P.C. Dwadash Shreni and Company (P) Ltd. , Aligarh , Allahabad, Patna

[5]. LUSTRENIK, L. A.and SOBOLEV, V.J. :Elements of Functional Analysis, Hindustan Publishing Corporation, India , (1961) . Cited by 2

[6]. MUNROE, M. E. : Introduction to measure and integration, Addison-Wesley Publishing Company, Inc., Reading Mass, U.S.A. (1959) . Cited by 399

[7]. NATANSON , I.P. : Theory of Functions of a real variable, Frederic Umgar, New York, (1955) Cited by 1474

Journal Papers:

[8]. S.N.Prasad, Analogues of some result on sequence spaces for function spaces (Quart . journal of Math , Oxford , 1960)

[9]. Amar Nath Kumar, On parametric and projective convergence in dual function spaces, (Aryabhatta research journal of physical science ,Ara,Bhojpur,Bihar),ISSN-0972-2432 Vol-16, Nos.-1-2 (2013), Page 11 to 13. 